\title{
Habitual tea consumption and 5-year incident metabolic syndrome among older adults: a community-based cohort study
}

Xing-Xuan Dong ${ }^{1 \dagger}$, Rui-Rui Wang ${ }^{1 \dagger}$, Jie-Yu Liu', Qing-Hua Ma² and Chen-Wei Pan ${ }^{1 *}$

\begin{abstract}
Background: The effect of tea consumption on metabolic syndrome (MetS) remains controversial. The objective of this study is to examine the prospective association of tea consumption with 5-year incident MetS among aged population in China.

Methods: This analysis included 3005 Chinese adults aged 60 years or older who were free of MetS at baseline examination. MetS was defined according to the National Cholesterol Education Program-Adult Treatment Panel III. Information regarding tea consumption was collected via an interviewer-administrated questionnaire. The prospective associations between tea consumption at baseline and 5-year incident MetS, as well as its individual components, were assessed by multiple logistic regression models.
\end{abstract}

Results: Of the 3005 participants free of MetS at baseline, 406 participants (cumulative incidence: 13.5\%) developed MetS at the 5-year follow-up examination. In multiple logistic regressions, 5-year cumulative incidence of MetS was found to be higher in those who drank tea more than 5 times per week as compared with non-habitual drinkers $(\mathrm{OR}=1.38,95 \% \mathrm{Cl}: 1.05-1.82 ; P=0.02)$. This relationship still existed in men $(\mathrm{OR}=1.43,95 \% \mathrm{Cl}: 1.00-2.01 ; P=0.05)$ when stratified by gender. Among the five major components of MetS, low high-density lipoprotein cholesterol was observed in men, while high body mass index, elevated blood pressure and the presence of diabetes mellitus were significant in women.

Conclusions: High-frequent tea consumption increased the risk of MetS among older Chinese adults. These findings may add novel knowledge to the current studies regarding the controversial effect of tea consumption on cardiovascular and metabolic health among the aged population.

Keywords: Metabolic syndrome, Tea consumption, Older adults, Cohort study

\section{Background}

Metabolic syndrome (MetS) is a global health concern associated with cardiovascular diseases, common cancers, increased mortality and great socieconomic burden,

\footnotetext{
*Correspondence: pcwonly@gmail.com

${ }^{\dagger}$ Xing-Xuan Dong and Rui-Rui Wang contributed equally to the work presented here and therefore should be considered equivalent authors. ${ }^{1}$ School of Public Health, Medical College of Soochow University, 199 Ren Ai Road, Suzhou 215123, China

Full list of author information is available at the end of the article
}

particularly in aged populations. The prevalence of MetS has increased over recent decades, reaching alarming rates worldwide [1, 2]. In 2016, the World Health Organization estimated that one-fifth of the world's adult population will develop MetS during their lifespan and the impact on their wellbeing and the health systems is tremendous. Thus, understanding its modifiable risk factors is crucial for formulating population-based intervention strategies for this condition. 
Tea, the dried leaves of the plant Camellia sinensis, is a popular beverage worldwide. Recent evidence has suggested that tea consumption was associated with the risk of MetS but the effect seemed to be controversial. While some studies concluded that tea consumption is protective for MetS [3, 4], others reported non-significant associations [5, 6]. Furthermore, one study found that frequent consumption of tea increased the risk of MetS in rural China [7].

China is the most populous country all over the world and may have the greatest burden of MetS [8]. It is estimated that one-third of the Chinese adults are affected by MetS [9]. Meanwhile, tea, especially green tea, is very popular among middle-aged to elderly Chinese adults. Suzhou is one of the most important green tea producing areas in China, where tea drinking is a common lifestyle habit among general populations. In addition, the existing conflicting associations of tea and MetS are likely due to the lack of longitudinal study design. The purpose of this study is to explore the prospective associations between tea consumption and 5-year incidence of MetS in a community-based cohort study on older adults in Suzhou of China. The findings might be important for formulating non-pharmacological strategies for supplementing the management of MetS and its associated complications, especially in areas where tea drinking is a widely accepted cultural practice.

\section{Materials and methods}

\section{Study population}

The Weitang Geriatric Diseases study is a communitybased study conducted in Weitang town among older adults aged 60 years or older in Suzhou, China. Detailed information about the study has been published elsewhere [10-13]. In brief, participants in the Weitang town of Suzhou were invited via invitation letters and were screened according to local official records. After excluding migrated residents and participants who had been living there less than 6 months, we enrolled 5613 adults who were considered to be eligible to participate. In 2014, 4611 eligible adults attended the baseline clinical examinations, among whom 4579 had complete data on interviewer-administered questionnaires including an Abbreviated Mental Test (AMT), underwent anthropometric examinations, and blood sample analyses. Five years later, these 4579 participants with complete data in the baseline study were invited to participate in the follow-up examination. If necessary, our study team conducted home visits or revisits to participants who were absent at the follow-up examinations to encourage participation. Those who moved away and did not provide updated contact information, refused to participate, or died before commencement of the 5-year follow-up examination were excluded from the follow-up study. Death of previous participants was confirmed through official death registration forms.

For the recruited participants, informed consent forms in writing duly were collected before their examination. The baseline and follow-up examinations of the Weitang Geriatric Diseases study followed the tenets of the Helsinki Declaration and were approved by the Institutional Review Board of Soochow University.

\section{Clinical examinations}

The measurements of weight, height and blood pressure (BP) were performed using standardized methods. Body weight was measured to the nearest $0.1 \mathrm{~kg}$ without shoes. Height was measured in the standing position without shoes. Body mass index (BMI) was calculated as weight in kilograms divided by the square of height in meters $(\mathrm{kg} /$ $\mathrm{m}^{2}$ ). BP was measured 3 times or more after at least $5 \mathrm{~min}$ intervals of rest by an automatic blood pressure monitor (Dinamap model Pro Series DP110X-RW, 100V2; GE Medical Systems Information Technologies, Inc., Milwaukee, Wisconsin, United States) and the value of BP was calculated from the average of the last two readings. The levels of high-density lipoprotein cholesterol (HDLC), triglycerides (TG) and fasting plasma glucose (FPG) were determined from frozen blood samples collected and analyzed with the standard laboratory assays by laboratory technicians.

\section{Definition of MetS}

We followed the National Cholesterol Education Program-Adult Treatment Panel III to define MetS based on the following five conditions [14]: (a) BMI of $25 \mathrm{~kg} /$ $\mathrm{m}^{2}$ or more; (b) BP of $130 / 85 \mathrm{mmHg}$ or higher or on anti-hypertension medications; (c) blood TG $\geq 150 \mathrm{mg} /$ $\mathrm{dL}(1.7 \mathrm{mmol} / \mathrm{L})$; (d) blood HDL-C of lower than $40 \mathrm{mg} /$ $\mathrm{dL}$ in men and $50 \mathrm{mg} / \mathrm{dL}$ in women; (e) fasting plasma glucose $\geq 7.0 \mathrm{mmol} / \mathrm{L}$ or with a history of diabetes mellitus [15]. If an individual was affected by three out of the above five conditions, he or she was considered to have MetS [16].

\section{Measurement of tea consumption}

Detailed information on tea consumption of the participants was collected at recruitment stage by a trained research assistant through the survey. Participants responded to the question "Do you usually drink tea?" Those responded "no" were identified as non-habitual tea drinkers. Habitual tea drinkers are defined by tea consumption of $120 \mathrm{~mL} / \mathrm{d}$ or more for at least 1 year. The frequency of tea drinking over the past 12 months was classified into "1-5 times/week" and "> 5 times/week", tea type was grouped into green tea and other tea since most 
of the habitual tea drinkers drank green tea in Suzhou, China. The duration of tea drinking was categorized into "1-15 years", "16-30 years" and "> 30 years". Detailed information regarding the cohort's tea drinking behaviors at baseline has been published elsewhere $[17,18]$.

\section{Covariates}

Participants' baseline information including socio-demographic characteristics (age, gender, education level, marital status, working status and monthly income), lifestyle-related habits (current smoking, alcohol consumption and physical activity) was collected in the baseline examinations. Marital status was defined as living "with" spouse or "without", education level was determined into "primary education or below" or "secondary education or above", and monthly income was made into three groups: "1000 Chinese Yuan (CNY) or less", "1001-3000 CNY" and "more than $3000 \mathrm{CNY}$ ".

\section{Statistical analysis}

Continuous and categorical variables were presented as mean \pm standard deviation (SD) and frequency (percentage), respectively. Characteristics of participants who were habitual tea drinkers versus non-habitual tea drinkers were compared using Student $t$-test or Chi-square test for continuous and categorical variables, respectively. Linear trends of individual MetS components across the frequency of tea consumption (0 times/week, 1-5 times/ week, $>5$ times/week) were investigated by Chi-square trend test. Two multiple logistic regression models were established to assess the relationship between tea consumption at baseline and the 5-year cumulative incidence of MetS as well as its individual components including high BP, high BMI, diabetes mellitus, low HDL-C and high TG, adjusting for potential confounders. Sensitivity analysis was used to select the most influential confounding factors. Model 1 adjusted for age and gender. Model 2 additionally controlled for initial BMI, educational level, monthly income and marriage status. Effect estimates including odds ratios (ORs) and their corresponding 95\% confidence intervals (CIs) were presented. Genderstratified analysis was also performed based on the same models. Interaction effects (different combinations of tea consumption and age, gender, education, socioeconomic status and other lifestyle habits such as smoking status, alcohol intake and physical activity) were investigated and excluded if the effects were not statistical significant. A two-sided $P$-value of less than 0.05 was considered statistically significant. Statistical analyses were performed using the SPSS version 21.0 (SPSS Inc., Chicago, IL, USA).

\section{Results}

Among the 4579 participants with complete data in the baseline survey, we excluded 183 (4.0\%) participants who had died prior to the 5-year follow-up examination and 526 (11.5\%) participants who were lost to follow-up or deceased. The mean age of the participants at follow-up examinations was $70.7 \pm 5.7$ years (range: $65.0-76.4$ years). Among the 3870 participants who successfully attended the follow-up examinations, 779 with MetS at baseline and 86 who had missing information for MetS features in the follow-up period were excluded as well, leaving 3005 participants available for the current prospective analyses ultimately. In the remained 3005 participants free of MetS at baseline survey, there were 1077 habitual and 1928 non-habitual tea drinkers, respectively. Table 1 compares the baseline characteristics between habitual and non-habitual tea drinkers. In general, habitual tea drinkers were more likely to be men $(P<0.001)$, current smokers $(P<0.001)$, alcohol drinkers $(P<0.001)$, and better educated $P<0.001)$. They also tended to live with spouse $(P<0.001)$, have higher income $(P<0.001)$, and have more physical activity $(P<0.001)$.

Among the 3005 participants free of MetS at baseline, 406 participants (cumulative incidence: 13.5\%) developed MetS at the 5-year follow-up examination. We found that increased frequency of tea consumption was associated with decreased systolic BPs and decreased HDL-C levels (all $P$ for trend $<0.001$ ). Other components such as BMI, diastolic BPs, blood FPG and TG were not significantly related to the frequency of tea consumption.

The results of sensitivity analysis are presented in Fig. 1. We selected age, gender, initial BMI, educational level, monthly income and marriage status as confounding factor to be controlled. Prospective associations between tea consumption and the incident MetS were further examined and the results are shown in Table 2. After adjusting for baseline demographics such as initial BMI, educational level, monthly income and marriage status (Model 2), habitual drinkers had a 31\% increased risk of developing MetS during the 5-year follow-up period compared with non-habitual drinkers $(\mathrm{OR}=1.31$, 95\% CI: 1.01-1.71; $P=0.04)$. However, the significant association was not observed either in men $(\mathrm{OR}=1.38$, 95\%CI: $0.98-1.95 ; P=0.06)$ or women $(\mathrm{OR}=1.17,95 \% \mathrm{CI}$ : $0.76-1.79 ; P=0.48)$. Correspondingly, 5-year cumulative incidence of MetS was found to be higher in those who drank tea more than 5 times per week $(\mathrm{OR}=1.38$, 95\% CI: $1.05-1.82 ; P=0.02)$ as compared with nonhabitual drinkers. This relationship still persisted in men $(\mathrm{OR}=1.43,95 \% \mathrm{CI}: 1.00-2.01 ; P=0.05)$ but was not significant in women $(\mathrm{OR}=1.28,95 \% \mathrm{CI}$ : $0.79-2.08 ; P=0.32)$ when stratified by gender. The association between green tea consumption and incident MetS was non-significant 
Table 1 Characteristics of study participants according to tea consumption habits

\begin{tabular}{|c|c|c|c|c|}
\hline Characteristic & All persons $(n=3005)$ & $\begin{array}{l}\text { Non-habitual tea drinkers } \\
(n=1928)\end{array}$ & $\begin{array}{l}\text { Habitual tea drinkers } \\
(n=1077)\end{array}$ & $P$ value \\
\hline \multicolumn{5}{|l|}{ Baseline characteristics } \\
\hline Gender (women), n (\%) & $1458(48.5)$ & $1297(67.3)$ & $161(14.9)$ & $<0.001$ \\
\hline Age, mean(SD), years & $67.3(5.7)$ & $67.5(5.9)$ & $67.0(5.4)$ & 0.01 \\
\hline Living with spouse, n (\%) & $2506(83.4)$ & 1567(81.3) & $939(87.2)$ & $<0.001$ \\
\hline Primary and below education, $\mathrm{n}$ & 2597(86.4) & 1766(91.6) & $831(77.2)$ & $<0.001$ \\
\hline Monthly income, n (\%) & & & & $<0.001$ \\
\hline$\leq 1000 \mathrm{CNY}$ & 1682(56.0) & 1177(61.0) & $505(46.9)$ & \\
\hline $1001-3000$ CNY & 1101(36.6) & $656(34.0)$ & $445(41.3)$ & \\
\hline$>3000 \mathrm{CNY}$ & $222(7.4)$ & $95(4.9)$ & $127(11.8)$ & \\
\hline Current smoking, n (\%) & $844(28.1)$ & $271(14.1)$ & $573(53.2)$ & $<0.001$ \\
\hline Alcohol consumption, n (\%) & $749(24.9)$ & $252(13.1)$ & $497(46.1)$ & $<0.001$ \\
\hline Physical activity, n (\%) & $1273(42.4)$ & 754(39.1) & $519(48.2)$ & $<0.001$ \\
\hline Working, n (\%) & 1135(37.8) & $671(34.8)$ & $464(43.1)$ & $<0.001$ \\
\hline $\mathrm{SBP}$, mean(SD), mmHg & 142.4(19.6) & 143.9(19.6) & 139.6(19.3) & $<0.001$ \\
\hline $\mathrm{DBP}$, mean(SD), mmHg & $84.9(11.3)$ & $84.8(11.3)$ & $85.1(11.3)$ & 0.46 \\
\hline $\mathrm{BMl}$, mean(SD), kg/m² & $22.9(4.9)$ & $22.8(5.8)$ & $23.1(2.4)$ & 0.11 \\
\hline $\mathrm{HDL}-\mathrm{C}$, mean(SD), mmol/L & $1.6(0.4)$ & $1.6(0.4)$ & $1.5(0.4)$ & $<0.001$ \\
\hline $\mathrm{TG}$, mean(SD), mmol/L & $1.1(0.5)$ & $1.1(0.5)$ & $1.1(0.6)$ & 0.44 \\
\hline $\mathrm{FPG}$, mean(SD), mmol/L & $5.4(0.9)$ & $5.4(0.9)$ & $5.4(0.9)$ & 0.24 \\
\hline History of hypertension, n (\%) & $1481(49.3)$ & $932(48.3)$ & $549(51.0)$ & 0.17 \\
\hline History of diabetes mellitus, n (\%) & $120(4.0)$ & $76(3.9)$ & $44(4.1)$ & 0.85 \\
\hline \multicolumn{5}{|l|}{ Follow-up characteristics } \\
\hline $\mathrm{SBP}$, mean(SD), $\mathrm{mmHg}$ & $148.9(21.0)$ & $150.1(21.2)$ & 146.6(20.4) & $<0.001$ \\
\hline DBP, mean(SD), mmHg & $82.1(11.5)$ & $81.6(11.5)$ & $83.1(11.3)$ & 0.001 \\
\hline $\mathrm{BMI}$, mean(SD), kg/m² & 23.6(3.6) & 23.5(3.8) & 23.8(3.0) & 0.05 \\
\hline $\mathrm{HDL}-\mathrm{C}$, mean(SD), mmol/L & $1.6(0.4)$ & $1.6(0.4)$ & $1.5(0.4)$ & $<0.001$ \\
\hline $\mathrm{TG}$, mean(SD), mmol/L & $1.2(0.7)$ & $1.3(0.7)$ & $1.2(0.7)$ & 0.27 \\
\hline FPG, mean(SD), mmol/L & $5.7(1.0)$ & $5.6(0.9)$ & $5.7(1.1)$ & 0.17 \\
\hline Metabolic syndrome, n (\%) & $406(13.5)$ & 263(13.6) & 143(13.3) & 0.78 \\
\hline
\end{tabular}

SD Standard deviation, CNY Chinese Yuan, SBP Systolic blood pressure, DBP Diastolic blood pressure, BMI Body mass index, HDL-C High-density lipoprotein cholesterol TG Triglycerides, FPG Fasting plasma glucose

in all study participants $(\mathrm{OR}=1.27,95 \% \mathrm{CI}: 0.97-1.67$; $P=0.09)$, men $(\mathrm{OR}=1.26$, 95\% CI: $0.91-1.76 ; P=0.17)$ and women $(\mathrm{OR}=1.22$, 95\% CI: $0.75-1.99 ; P=0.42)$. Participants who drank tea for 16 to 30 years were more likely to develop MetS than non-habitual drinkers $(\mathrm{OR}=1.53,95 \% \mathrm{CI}: 1.02-2.28 ; P=0.04)$. The results were significant in neither men $(\mathrm{OR}=1.39,95 \% \mathrm{CI}$ : 0.85 $2.28 ; P=0.19)$ nor women $(\mathrm{OR}=1.79,95 \% \mathrm{CI}$ : $0.87-3.71$; $P=0.11$ )

Figure 2 illustrates the prospective associations between tea consumption and individual components of MetS. No significant relationship was observed between the tea consumption at baseline and incident high BP, high BMI, diabetes mellitus or low HDL-C levels. The presence of tea consumption at baseline was related to high TG in both Model 1 (OR $=1.34,95 \%$
CI: $1.03-1.73 ; P=0.03)$ and Model $2(\mathrm{OR}=1.32,95 \%$ CI: $1.02-1.72 ; P=0.04)$ among the participants, and the significant association still exist in men after stratified by gender $(\mathrm{OR}=1.47,95 \% \mathrm{CI}: 1.03-2.10 ; P=0.03)$.

Considering that tea consumption was much more frequent in men than women, we performed a genderstratified analysis and the results are depicted in Fig. 1. Gender could be a possible influencing factor on the associations between habitual tea consumption and individual components of MetS. We found that low HDL-C levels were related to tea consumption in men $(\mathrm{OR}=2.03,95 \% \mathrm{CI}: 1.11-3.69 ; P=0.02)$. However, in women, tea consumption was positively associated with high BMI and elevated BP. Additionally, tea consumption was related with the presence of diabetes mellitus among women in Model $2(\mathrm{OR}=2.02,95 \% \mathrm{CI}$ : 


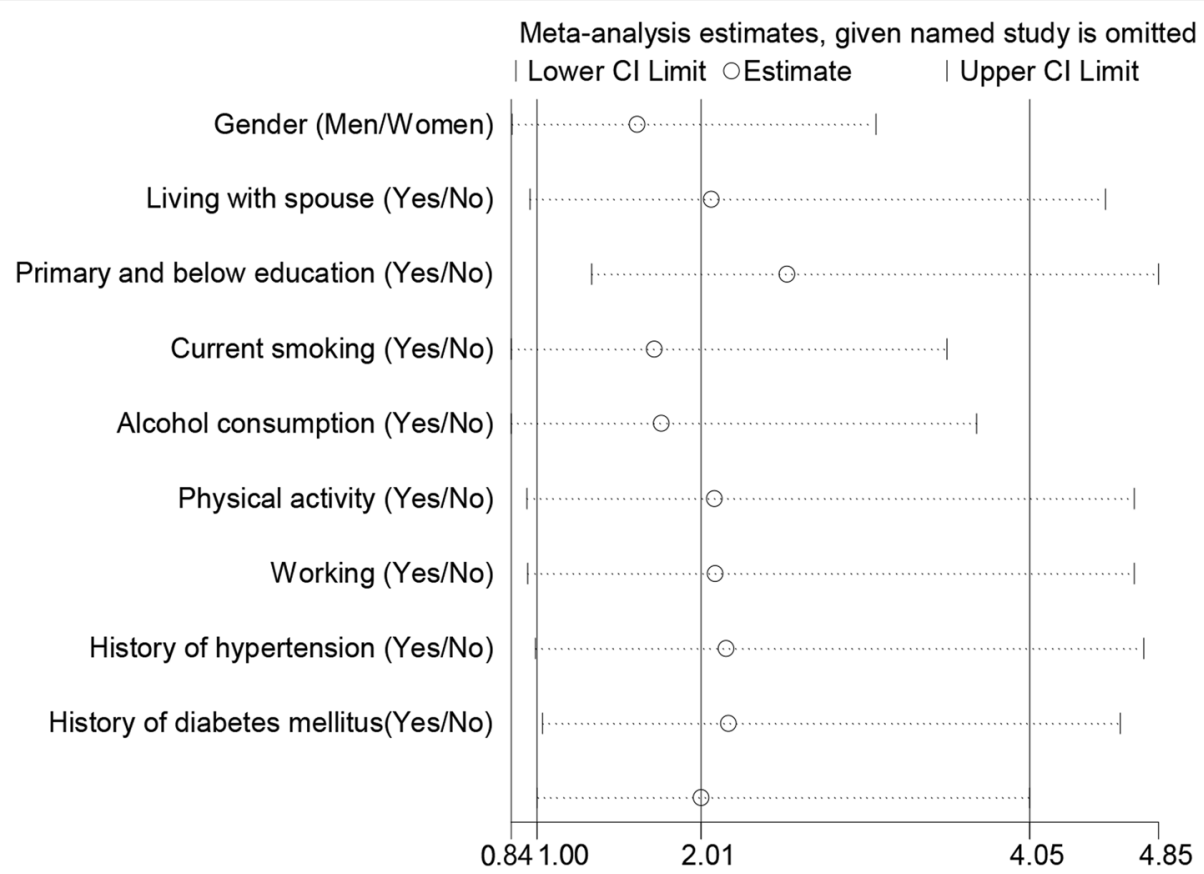

Fig. 1 Sensitivity analysis of different characteristics and tea consumption habits of the study participants

1.07-3.83; $P=0.03)$. The interaction effects between tea consumption and other variables were not observed.

\section{Discussion}

In this 5-year prospective study on older Chinese adults, increased risk of developing MetS was observed in those who drank tea more than 5 times per week as compared with non-habitual drinkers. A dose-response relationship was also observed with increased frequency of green tea consumption being associated with a higher risk of MetS. In addition, the association of tea consumption and individual components of MetS varied between men and women. These findings added novel knowledge to the current literatures regarding the controversial effect of tea consumption on cardiovascular and metabolic health among the elderly.

The main finding of our study was different from most studies, which reported beneficial effects of tea consumption for MetS and some of its individual components $[3,4,19]$. A systematic review and meta-analysis summarized the findings of 6 observational studies and demonstrated that tea consumption were associated with a reduced risk of MetS [20]. Meanwhile, null associations were also reported in some other studies $[5,6$, 21]. Weak relation of tea with MetS pointed towards the potential importance of composition of polyphenols and the types of tea consumed, and these observations needs to be confirmed in well-designed cohort studies. Based on the results of our analysis, there is, however, an increased risk of developing MetS in older adults who drank tea more frequently. Particularly, we found that habitual drinkers who drank tea more than 5 times per week were more susceptible to MetS. In addition, the relationship remained significantly different among men. In our study, women were less likely to be habitual tea drinkers compared with men, which reduced the sample size of women and may mask the association between tea consumption and MetS. Moreover, genderstratified analysis may weaken the statistical power as the sample size was reduced by nearly half, making the effect of tea consumption on MetS insignificant either in men or women. Previous results indicated the deleterious effects of tea consumption, which was independently associated with an increased risk of kidney stones in Northern Chinese [22]. Concentrated herbal extracts from green tea may increase the burden of liver metabolism and may not be free of adverse effects under certain circumstances [23]. Additionally, about $80-85 \%$ of oxalate in diets of Chinese individuals was derived from tea and coffee [24], as a result, heavy tea-drinking habits will increase the excretion of urinary oxalic acid, which may lead to kidney stone formation, and eventually increase the burden of circulation. Similar results were not found when stratified by tea drinking duration. We considered that the information which relied on the subjective judgment and recall of the participants may lead to misclassification and information bias, thus may have distorted the effect estimates of tea consumption on MetS. In 


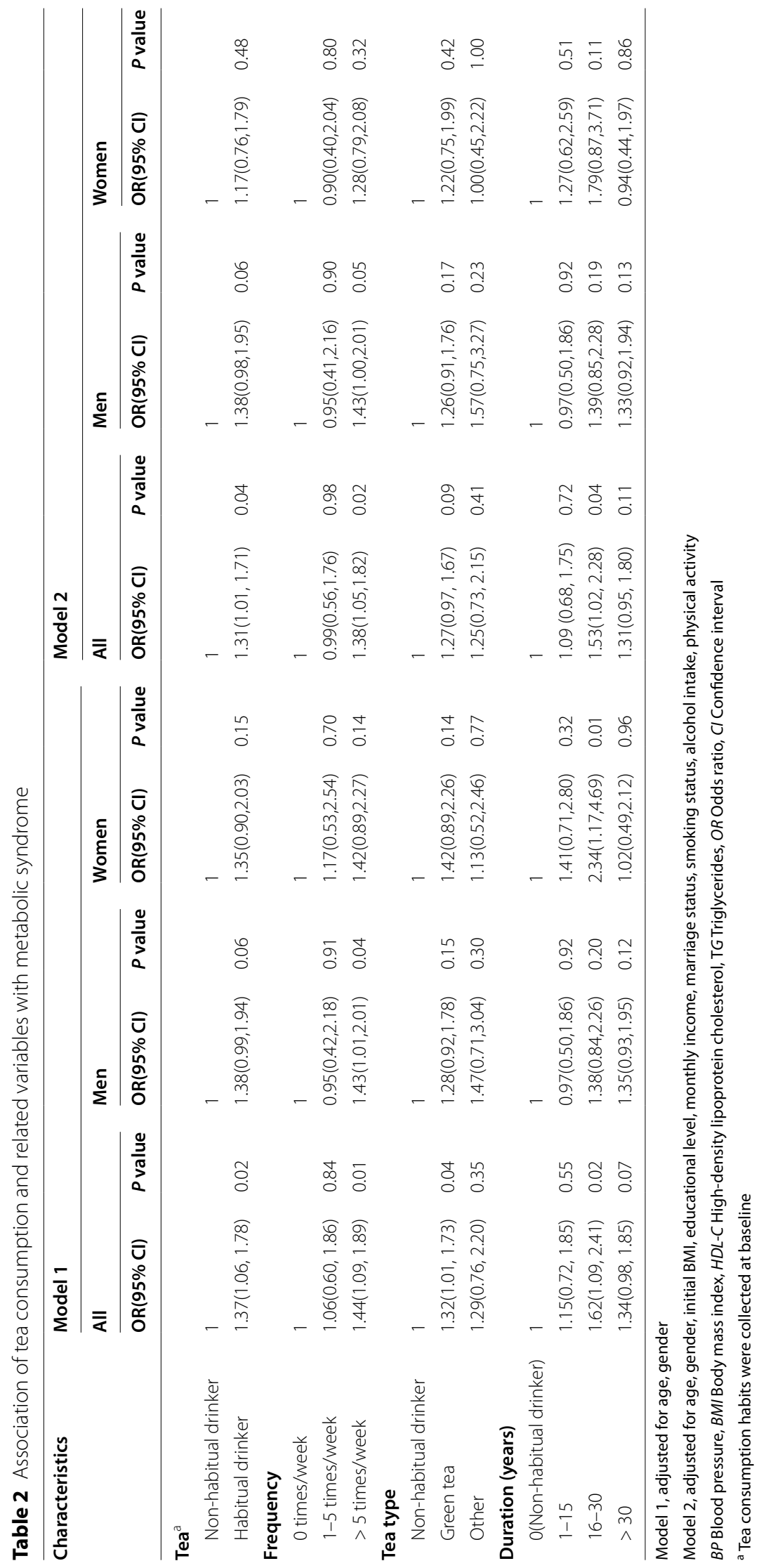




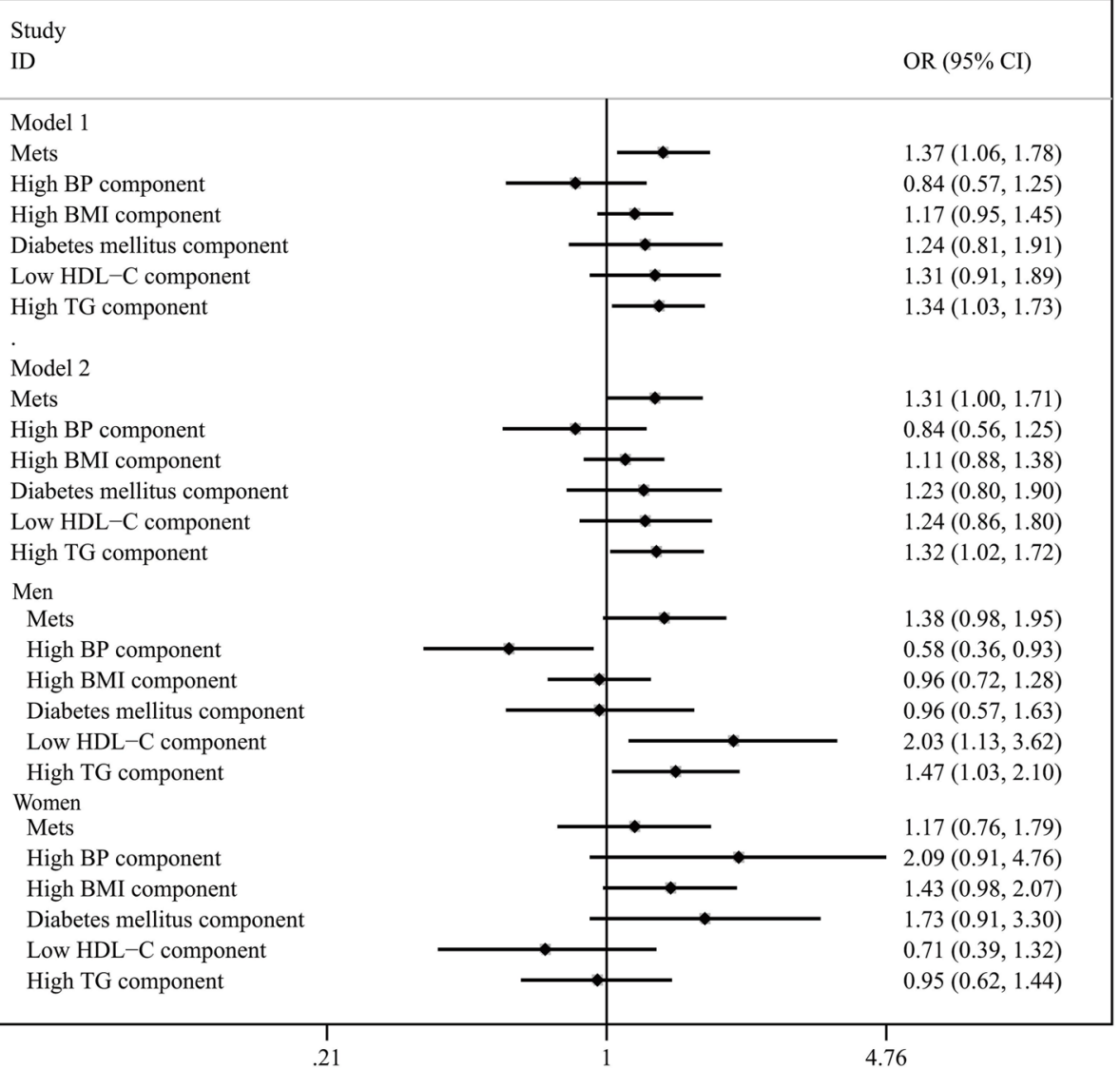

Fig. 2 Risk of developing metabolic syndrome and its components according to tea consumption habits. BP, blood pressure; BMI, body mass index; $\mathrm{HDL}-\mathrm{C}$, high-density lipoprotein cholesterol; $\mathrm{TG}$, triglycerides; OR, odds ratio; $\mathrm{Cl}$, confidence interval. * Tea consumption habits were collected at baseline. Model 1, adjusted for age, gender. Model 2, adjusted for age, gender, initial BMI, educational level, monthly income and marriage status

addition, perennial tea drinking habits may change due to some influential factors, such as health education and disease. What's more, the information about the preparation method of tea (brewing or boiling) and the container of tea drinking was not available, so the serving size of tea may not be described accurately. Moreover, we found that habitual drinkers were more likely to be alcohol consumers, as shown in Table 1. The baseline characteristics of the 709 participants who did not enter the analysis were not different from those who entered the analysis. In rural China, having tea after taking alcohol is thought to be able treat effects of drunkenness or hangover. By leading the toxic acetaldehyde converted from alcohol to the kidney along with tea absorption before it decomposes, tea consumption after drinking could damage the kidney and finally disrupt normal blood flow [25]. Further studies are warranted to validate these hypotheses.

With regards to the association between tea consumption and individual components of MetS, we observed a gender difference. Low HDL-C levels were related to tea consumption in men while tea consumption was positively associated with high BMI, elevated BP and the presence of diabetes mellitus in women. The mechanisms underlying this phenomenon might be attributed to different lifestyles between men and women. For instance, women are significantly less physically active than men and obese women are most functionally impaired, and had lower perceived behavioral control toward physical activity [26]. Apart from this, one survey also found that men were more likely to smoke and drink alcohol [27]. Physical activity, smoking and alcohol intake are wellestablished risk factors for MetS and thus may modify the tea-MetS association between men and women.

The public health implication of our findings needs to be discussed. Tea consumption is traditionally considered to be a promising non-pharmacological strategy for supplementing the management of hypertension, obesity or diabetes, especially in places where tea drinking is a widely accepted cultural practice. In the era of rapid population aging and high prevalence of chronic diseases, tea drinking is regarded as an inexpensive and applicable dietary practice, which may be clinically relevant and 
show its public health importance. However, our study indicated that these beneficial effects need carefully reconsideration, especially when the drinkers are restricted to older adults. Some other aspects such as frequency of drinking, way of preparations and time for drinking should also be taken into consideration. The findings might be important for formulating non-pharmacological strategies for supplementing the management of MetS.

Our study is a community-based cohort study with a large sample size and a reasonable follow-up rate. Therefore, we provided a more cogent evidence than case-control or cross-sectional studies. Several limitations should also be acknowledged. First, the included participants were restricted to older Chinese adults who lived in the eastern part of China with proportionately high prevalence and incidence of MetS and extensive extrapolation of the findings to other populations needs further clarification. Second, tea consumption was self-reported and thus may result in recall biases, especially in older adults with cognitive decline. Finally, although we had controlled for a wide range of confounders, the possibility of unmeasured and residual confounding such as chronic diseases or medication treatment, the type of diet and dietary patterns, and whether malnutrition was involved that might have contributed to the risk of MetS, which might distort the associations observed in this study. Diet in Jiangnan region is healthier than that in other regions. On the one hand, residents are used to cooking with squeezed rapeseed oils, which is rich in Omega- 6 and contributes to reducing total mortality [28-30]. On the other hand, due to the sufficient rainfall and fertile plains, Jiangnan residents often eat abundant aquatic products and fresh vegetables and fruits [31]. More studies are required to confirm the findings from this current study.

\section{Conclusions}

In conclusion, increased risk of developing MetS was observed in high-frequent tea drinkers among older adults. Further well-designed cohort studies with more precise measurement of tea consumption and long-term follow-up duration are warranted to replicate our findings and the mechanisms underlying the adverse effect of tea consumption needs to be elucidated.

\section{Abbreviations}

MetS: Metabolic syndrome; BMI: Body mass index; HDL-C: High-density lipoprotein cholesterol; TG: Triglycerides; FPG: Fasting plasma glucose.

\section{Acknowledgements}

Not applicable.

\section{Authors' contributions}

Xing-Xuan Dong: Methodology, Investigation, Resources, Data curation, Writing - review \& editing, Visualization. Rui-Rui Wang: Conceptualization, Methodology, Software, Validation, Investigation, Resources, Writing - review \& editing, Visualization. Jie-Yu Liu: Conceptualization, Methodology, Software, Validation, Formal analysis, Investigation, Resources, Writing - original draft. Qing-Hua Ma: Conceptualization, Methodology, Supervision. Chen-Wei Pan: Conceptualization, Methodology, Resources, Supervision, Project administration, Funding acquisition. The author(s) read and approved the final manuscript.

\section{Funding}

This study was supported by the Science and Technology Bureau of Xiangcheng District in Suzhou, China under grant no. XJ201706, the Health Commission of Suzhou under grant no. GSWS2019090, the Tang Scholar of Soochow University and the Priority Academic Program Development of Jiangsu Higher Education Institutions (PAPD).

\section{Availability of data and materials}

The datasets used and/or analyzed during the current study are available from the corresponding author on reasonable request.

\section{Declarations}

\section{Ethics approval and consent to participate}

For the recruited participants, informed consent forms in writing duly were collected before their examination. This study was conducted comply with the tenets of the Helsinki Declaration, and with the approval of the Institutional Review Board of Soochow University.

\section{Consent for publication}

Not applicable.

\section{Competing interests}

The authors declare that they have no competing interests.

\section{Author details}

${ }^{1}$ School of Public Health, Medical College of Soochow University, 199 Ren Ai Road, Suzhou 215123, China. ${ }^{2}$ The 3rd People's Hospital of Xiangcheng District, Suzhou, China.

Received: 2 April 2021 Accepted: 8 December 2021

Published online: 19 December 2021

\section{References}

1. Gebreyes YF, Goshu DY, Geletew TK, Argefa TG, Zemedu TG, Lemu $K A$, et al. Prevalence of high bloodpressure, hyperglycemia, dyslipidemia, metabolic syndrome and their determinants in Ethiopia: evidences from the national NCDs STEPS survey, 2015. PLoS One. 2018;13(5):e0194819.

2. Chowdhury MZI, Anik AM, Farhana Z, Bristi PD, Abu Al Mamun BM, Uddin MJ, et al. Prevalence of metabolic syndrome in Bangladesh: a systematic review and meta-analysis of the studies. BMC Public Health. 2018;18(1):308.

3. Grosso G, Stepaniak U, Micek A, Topor-Mądry R, Pikhart H, Szafraniec K, et al. Association of daily coffee and tea consumption and metabolic syndrome: results from the Polish arm of the HAPIEE study. Eur J Nutr. 2015;54(7):1129-37.

4. Chang CS, Chang YF, Liu PY, Chen CY, Tsai YS, Wu CH. Smoking, habitual tea drinking and metabolic syndrome in elderly men living in rural community: the Tianliao old people (TOP) study 02. PLoS One. 2012;7(6):e38874.

5. Takami $\mathrm{H}$, Nakamoto $\mathrm{M}$, Uemura $\mathrm{H}$, Katsuura S, Yamaguchi M, Hiyoshi M, et al. Inverse correlation between coffee consumption and prevalence of metabolic syndrome: baseline survey of the Japan Multi-Institutional Collaborative Cohort (J-MICC) Study in Tokushima, Japan. J Epidemiol. 2013:23(1):12-20

6. Micek A, Grosso G, Polak M, Kozakiewicz K, Tykarski A, Puch Walczak A, et al. Association between tea and coffee consumption and prevalence 
of metabolic syndrome in Poland - results from the WOBASZ II study (2013-2014). Int J Food Sci Nutr. 2018;69(3):358-68.

7. Yu S, Guo X, Yang H, Zheng L, Sun Y. An update on the prevalence of metabolic syndrome and its associated factors in rural northeast China. BMC Public Health. 2014;14:877.

8. Liu X, Xu W, Cai H, Gao YT, Li H, Ji BT, et al. Green tea consumption and risk of type 2 diabetes in Chinese adults: the Shanghai women's health study and the Shanghai men's health study. Int J Epidemiol. 2018:47(6):1887-96.

9. Lu J, Wang L, Li M, Xu Y, Jiang Y, Wang W, et al. Metabolic syndrome among adults in China: the 2010 China noncommunicable disease surveillance. J Clin Endocrinol Metab. 2017;102(2):507-15.

10. Yang XJ, Tian S, Ma QH, Sun HP, Xu Y, Pan CW. Leukocyte-related parameters in older adults with metabolic syndrome. Endocrine. 2020;68(2):312-9.

11. Liu JH, Qian YX, Ma QH, Sun HP, Xu Y, Pan CW. Depressive symptoms and metabolic syndrome components among older Chinese adults. Diabetol Metab Syndr. 2020;12:18.

12. Liu JH, Zhang YJ, Ma QH, Sun HP, Xu Y, Pan CW. Elevated blood neutrophil to lymphocyte ratio in older adults with cognitive impairment. Arch Gerontol Geriatr. 2020;88:104041.

13. Liu JH, Ma QH, Sun HP, Xu Y, Pan CW. Depressive symptom as a mediator of the influence of self-reported sleep quality on falls: a mediation analysis. Aging Ment Health. 2021;25(4):728-33.

14. Expert Panel on Detection, Evaluation, and Treatment of High Blood Cholesterol in Adults. Executive summary of the third report of the National Cholesterol Education Program (NCEP) expert panel on detection, evaluation, and treatment of high blood cholesterol in adults (adult treatment panel III). JAMA. 2001;285(19):2486-97.

15. Sabanayagam C, Wang JJ, Mitchell P, Tan AG, Tai ES, Aung T, et al. Metabolic syndrome components and age-related cataract: the Singapore Malay eye study. Invest Ophthalmol Vis Sci. 2011;52(5):2397-404.

16. Qian YX, Liu JH, Ma QH, Sun HP, Xu Y, Pan CW. Associations of sleep durations and sleep-related parameters with metabolic syndrome among older Chinese adults. Endocrine. 2019;66(2):240-8.

17. Gu YJ, He CH, Li S, Zhang SY, Duan SY, Sun HP, et al. Tea consumption is associated with cognitive impairment in older Chinese adults. Aging Ment Health. 2018;22(9):1232-8.

18. Yin JY, Duan SY, Liu FC, Yao QK, Tu S, Xu Y, et al. Blood pressure is associated with tea consumption: a cross-sectional study in a rural, elderly population of Jiangsu China. J Nutr Health Aging. 2017;21(10):1151-9.

19. Vernarelli JA, Lambert JD. Tea consumption is inversely associated with weight status and other markers for metabolic syndrome in US adults. Eur J Nutr. 2013;52(3):1039-48.

20. Marventano S, Salomone F, Godos J, Pluchinotta F, Del Rio D, Mistretta $A$, et al. Coffee and tea consumption in relation with non-alcoholic fatty liver and metabolic syndrome: a systematic review and meta-analysis of observational studies. Clin Nutr. 2016;35(6):1269-81.

21. Hino A, Adachi H, Enomoto M, Furuki K, Shigetoh Y, Ohtsuka M, et al. Habitual coffee but not green tea consumption is inversely associated with metabolic syndrome: an epidemiological study in a general Japanese population. Diabetes Res Clin Pract. 2007;76(3):383-9.

22. Wu ZB, Jiang T, Lin GB, Wang YX, Zhou Y, Chen ZQ, et al. Tea consumption is associated with increased risk of kidney stones in northern Chinese: a cross-sectional study. Biomed Environ Sci. 2017;30(12):922-6.

23. Gaeini Z, Bahadoran Z, Mirmiran P, Azizi F. Tea, coffee, caffeine intake and the risk of cardio-metabolic outcomes: findings from a population with low coffee and high tea consumption. Nutr Metab (Lond). 2019;16:28.

24. Gasińska A, Gajewska D. Tea and coffee as the main sources of oxalate in diets of patients with kidney oxalate stones. Rocz Panstw Zakl Hig. 2007;58(1):61-7.

25. Loria D, Barrios E, Zanetti R. Cancer and yerba mate consumption: a review of possible associations. Rev Panam Salud Publica. 2009;25(6):530-9.

26. Gretebeck KA, Sabatini LM, Black DR, Gretebeck RJ. Physical activity, functional ability, and obesity in older adults: a gender difference. J Gerontol Nurs. 2017:43(9):38-46.

27. Xia X, Yue W, Chao B, Li M, Cao L, Wang L, et al. Prevalence and risk factors of stroke in the elderly in northern China: data from the National Stroke Screening Survey. J Neurol. 2019;266(6):1449-58.
28. Jiao J, Liu G, Shin HJ, Hu FB, Rimm EB, Rexrode KM, et al. Dietary fats and mortality among patients with type 2 diabetes: analysis in two population based cohort studies. BMJ. 2019;366:14009.

29. Wang DD, Li Y, Chiuve SE, Stampfer MJ, Manson JE, Rimm EB, et al. Association of specific dietary fats with total and cause-specific mortality. JAMA Intern Med. 2016;176(8):1134-45.

30. Zong G, Liu G, Willett WC, Wanders AJ, Alssema M, Zock PL, et al. Associations between linoleic acid intake and incident type 2 diabetes among U.S. men and women. Diabetes Care. 2019;42(8):1406-13.

31. Wang J, Lin X, Bloomgarden ZT, Ning G. The Jiangnan diet, a healthy diet pattern for Chinese. J Diabetes. 2020;12(5):365-71.

\section{Publisher's Note}

Springer Nature remains neutral with regard to jurisdictional claims in published maps and institutional affiliations.

Ready to submit your research? Choose BMC and benefit from:

- fast, convenient online submission

- thorough peer review by experienced researchers in your field

- rapid publication on acceptance

- support for research data, including large and complex data types

- gold Open Access which fosters wider collaboration and increased citations

- maximum visibility for your research: over 100M website views per year

At BMC, research is always in progress.

Learn more biomedcentral.com/submissions 